\title{
Simultaneous reduction of MAD2 and BUBR1 expression induces mitotic spindle alterations associated with p53 dependent cell cycle arrest and death
}

\author{
Laura Lentini $^{1 *^{\dagger}}$, Desirèe Piscitello ${ }^{1 \dagger}$, Lorena Veneziano ${ }^{1}$ and Aldo Di Leonardo ${ }^{1,2}$ \\ 1 Department of Biological Chemical and Pharmaceutical Sciences and Technologies (STEBICEF), University of Palermo Viale delle Scienze, \\ Palermo 90128, Italy \\ 2 Center of Experimental OncoBiology (COBS), Via San Lorenzo, Palermo 90146, Italy
}

\begin{abstract}
Most human tumors are characterized by aneuploidy that is believed to be the consequence of chromosomal instability (CIN). The mechanism(s) leading to aneuploidy and the pathways that allow its tolerance are not completely understood. The Spindle Assembly Checkpoint (SAC) is a cellular surveillance mechanism working during mitosis, and alterations of genes that encode components of the SAC weakening the mitotic checkpoint, induce aneuploidy by chromosome mis-segregation. We induced aneuploidy in near-diploid tumor cells by simultaneous depletion of the SAC proteins MAD2 and BUBR1 by RNA interference in the attempt to gain further insight on the cellular responses to aneuploidy. Individual reduction of MAD2 and BUBR1 protein levels caused defective mitosis and aneuploidy, while co-depletion of MAD2 and BUBR1 caused cell cycle arrest and cell death in addition to aneuploidy. The simultaneous reduction of the two SAC proteins induced high percentage of hyperdiploid cells and p53 stabilization suggesting that hyperdiploidy could activate a p53 controlled pathway. The results indicate that p53 is required to induce cell cycle arrest and cell death when the mitotic checkpoint is strongly perturbed, thereby preventing aneuploid cell propagation.
\end{abstract}

Keywords: cell cycle; cell death; chromosomes

\section{Introduction}

Segregation of genetic material into 2 daughter cells during mitosis is a highly regulated event and errors during this phase of the cell cycle can generate cells with aberrant chromosome numbers (aneuploidy). Precise control of progression through mitosis is essential to maintain genomic stability and prevent aneuploidy (Ulrich, in press). Mutations/alterations in genes encoding mitotic regulators and defects in genes controlling centrosome numbers and tumor suppressors have been suggested as possible causes of aneuploidy (Lentini et al., 2002; Hernando et al., 2004; Fukasawa, 2005; Iovino et al., 2006; Lentini et al., 2006). Cells have evolved a surveillance mechanism, the Spindle Assembly Checkpoint (SAC), which prevents anaphase onset until all chromosomes are properly attached to the mitotic spindle ensuring that mitosis is faithfully accomplished preserving genome stability. The molecular components of the SAC include Mad1, Mad2, Bubr1, Bub1, Bub3, and Mps1 proteins. Alteration of MAD2 and BUBR1 expression may because of aneuploidy (Guo et al., 2012). In particular, human colorectal cancer cells (HCT116) and murine primary embryonic fibroblasts (MEFs), in which one MAD2 allele was deleted by homologous recombination, are highly aneuploid (Michel et al., 2001; Lentini et al., 2012). Haplo-insufficiency of MAD2 also increases the frequency of aneuploid tumors in a p53 background (Amato et al., 2009). Intriguingly, aneuploidy and tumorigenesis are also driven by hyperactivation of MAD2 (Sotillo et al., 2007; Schvartzman et al., 2011). The Bubr1 protein kinase, encoded by the BUB1B gene, is a crucial component for several processes controlling chromosome segregation during mitosis. Genetic mutations of the Bubrl kinase occur in the cancer susceptible disorder, mosaic variegated aneuploidy (MVA) (Matsuura et al., 2006), which is a rare disorder characterized by

*Corresponding author: e-mail: lauralentini@unipa.it

'These authors contributed equally. 
constitutional mosaic aneuploidies associated in most cases with premature chromatid separation (PCS), highlighting the key role of human Bubr1 in chromosome segregation (Matsuura et al., 2006). Bubr1 also functions in a positive regulatory loop with the tumor suppressor p53, thus enhancing apoptosis of polyploid cells (Shin et al., 2003). It facilitates phosphorylation and stabilization of p53 after mitotic spindle damage, as well as being a direct p53 transcriptional target (Aylon and Oren, 2011). Inhibition of Bubr1 in the presence of mitotic poisons accelerates polyploidy in a p53-null background, reinforcing the idea that reduction of chromosome segregation fidelity and acquisition of aneuploid tolerance work synergistically to promote cancer (Shin et al., 2003). However, the genomic imbalance caused by aneuploidy reduces fitness of euploid cells (Torres et al., 2008). Similarly, stable near-diploid tumor cells that become aneuploid after treatment with mitotic poisons are removed from the cell population within few generations (Thompson and Compton, 2008). These findings suggest that cancer cells must acquire the ability to overcome these barriers and that mutations triggering aneuploidy tolerance should accompany chromosome missegregation defects. The mechanisms leading to aneuploid tolerance in tumor cells are not yet completely understood. We found that partial depletion of MAD2 triggered aneuploidy in primary human fibroblasts followed by 553 stabilization that induced premature cellular senescence to avoid the deleterious effects of aneuploidy (Lentini et al., 2012). p53 could be responsible for the proliferative disadvantage of aneuploidy cells, as suggested also by $\mathrm{p} 53^{-1-}$ cells in which aneuploidy remains high. Only a fraction of MAD2 depleted HCT116 cells have whole chromosome aneuploidy associated with p53 activation (Li et al., 2010). Furthermore, the presence of aneuploid cells in vivo and primary MEFs showing aneuploidy has no proliferative disadvantage, despite having an intact p53 pathway, which suggest that p53 is activated in only some aneuploid cells (Zasadil et al., 2013).

Partial or complete inhibition of the mitotic kinase Aurora B results in chromosome misaggregation or failure of cytokinesis. To limit proliferation of aneuploid cells, a p53-mediated cell cycle arrest is induced, where p53 might be regulated in two separate ways, i.e. by being stabilized by inhibition of Aurora B itself, or by damage to the mitotic spindle apparatus. The stabilization is insufficient to activate p53 and a second pathway, the MAP kinase signaling pathway MAP3K4-p38, is required for transcriptional activation of p53. MAP3K4 might be activated by mitotic spindle disruption and trigger the signal to $38 \beta$. Presumably, $\mathrm{p} 38 \beta$ targets a transcriptional co-factor of p53 leading to full activity of p53 and induction of p21. The cell growth arrest induced by these pathways might prevent chromosomal instability and therefore suppress tumorigenesis (Ulrich, in press).
We triggered aneuploidy by weakening the SAC after post-transcriptional silencing of MAD2 and BUBR1 genes and investigated whether a p53 controlled pathway is activated in near-diploid tumor cells (HCT116) with a MIN (Microsatellite Instability) phenotype to avoid propagation of highly aneuploidy cells. Individual reduction of Mad2 and Bubr1 protein levels partially affected cell proliferation and caused mitotic spindle defects and aneuploidy in neardiploid HCT116 cells. Conversely, simultaneous Mad2 and Bubr1 depletion resulted in greater effects, leading to cell cycle arrest and high mortality.

\section{Materials and methods}

\section{Cells and cell culture}

Near-diploid HCT116 and HCT116 p53KO cells (a generous gift of Prof. B. Vogelstein, Ludwig Center for Cancer Genetics and Therapeutics, Johns Hopkins, Baltimore, MD, USA) with a MIN phenotype were cultured in D-MEM supplemented with $10 \%$ FBS (GIBCO, Invitrogen), 100 units $/ \mathrm{mL}$ penicillin and $0.1 \mathrm{mg} / \mathrm{mL}$ streptomycin in a humidified atmosphere of $5 \% \mathrm{CO}_{2}$ in air at $37^{\circ} \mathrm{C}$.

\section{RNA interference}

For the RNAi experiments, $2 \times 10^{5}$ HCT116 cells were plated in 6-well dishes, incubated at $37^{\circ} \mathrm{C}$ and transfected $24 \mathrm{~h}$ after plating with specific siRNA duplexes. Briefly, the siRNAs and the transfection reagent (Lipofectamine 2000, Invitrogen) were diluted separately in Opti-MEM (Invitrogen) mixed gently, and then incubated for $5 \mathrm{~min}$ at RT. After incubation the siRNAs and Lipofectamine 2000 (Invitrogen) were mixed gently, allowed to sit $30 \mathrm{~min}$ at RT to allow complex formation and added to the plates for $72 \mathrm{~h}$. After $6 \mathrm{~h}$ at $37^{\circ} \mathrm{C}$, the transfection medium was replaced with fresh medium. To silence genes of interest, post-transcriptionally, cells were transfected with siRNAs targeting MAD2 (siMAD2: 5'-AUACGGACUCACCUUUtt-3') (Michel et al., 2001), siRNAs targeting p53 (si p53: 5'-GCA UGA ACC GGA GGC CCC AUtt-3') (Frame et al., 2006) and BUBR1 (siBUBR1: 5'-GUCUCACAGAUUGCUGCCUtt-3') (Choi and Lee, 2008) at $60 \mathrm{nM}$. The control siRNA (siGFP: 5'-GGCUACGUCCAGGAGCGCACCtt- $3^{\prime}$ ) targets the Green-Fluorescent-Protein and was used at $60 \mathrm{nM}$. All siRNAs (21-nucleotide duplexes) were synthesized by Eurofins-MWG (Germany).

\section{Real time RT-PCR}

Primers to be used in Real time RT-PCR experiments were designed with Primer Express software (Applied Biosystems) choosing amplicons of $70-100 \mathrm{bp}$. The selected sequences were tested on public databases (BLAST) to 
confirm the identity of the genes. Total RNA was extracted from cells by using the RNAeasy Mini kit (Qiagen). RNA was reverse-transcribed in a final volume of $50 \mu \mathrm{L}$ using the High Capacity c-DNA Archive kit (Applied Biosystems) for $10 \mathrm{~min}$ at $25^{\circ} \mathrm{C}$ and $2 \mathrm{~h}$ at $37^{\circ} \mathrm{C}$. For each sample $2 \mu \mathrm{L}$ of cDNA, corresponding to $100 \mathrm{ng}$ of reverse transcribed RNA, were analyzed by Real time RT-PCR $\left(95^{\circ} \mathrm{C}\right.$ for $15 \mathrm{~s}, 60^{\circ} \mathrm{C}$ for $60 \mathrm{~s}$ repeated for 40 cycles), in quadruplicate, using the ABI PRISM 7300 instrument (Applied Biosystems). Real-time RT-PCR was done in $20 \mu \mathrm{L}$ comprising $1 \times$ Master Mix SYBR Green (Applied Biosystems) and $0.3 \mu \mathrm{M}$ of forward and reverse primers for: MAD2 (Fwd:5'-GCCGAGTTTTTCTCATTTGG-3'; Rev:5'-CCGATTCTTCCCACTTTTCA-3'), p53 (Fwd:5'-TTCGACATAGTGTGGTGGTGC$3^{\prime}, \quad$ Rev:5'-AGTCAGAGCCAACCTGAGGC- $\left.3^{\prime}\right), \quad$ p2 $1^{\text {waf } 1}$ (Fwd: 5'-CTGGAGACTCTCAGGGTCGA-3' Rev:5'-CGGATTAGGGCTTCCTCTTG-3'), BUBR1 (Fwd:5'-TACACTGGAAATGACCCTCTGGAT-3', Rev: 5'-TATATTATCGTTTTTCTCCTTGTAGTGCTT-3'), GAPDH (Fwd:5' CTCATGACCACAGTCCATGCC-3'; Rev:5'-GCCAATCCACAGTCTTCTGGGT- $\left.3^{\prime}\right)$. Data were analyzed by averaging quadruplicates $\mathrm{Ct}$ (cycle threshold). Levels of RNA expression were determined by using the SDS software version (Applied Biosystems) according to the 2- $\Delta \Delta \mathrm{ct}$ method. Levels of RNA expression of selected genes were normalized to the internal control GAPDH.

\section{Western blotting}

Protein concentration was measured using the Bio-Rad Protein Assay (Bio-Rad Laboratories). Proteins (50 $\mu \mathrm{g})$ were separated by $10 \%$ SDS-PAGE containing $0.1 \%$ SDS and transferred to Hybond-C nitrocellulose membranes (Amersham Life Science) by electroblotting (Lentini et al., 2014). The membranes were sequentially incubated with p53-DO1 (mouse, 1:1000), p21(mouse, 1:1000), Bubr1 (goat, 1:500), Mad2 (goat, 1:200) as primary antibodies (Santa Cruz,CA), and HRP-conjugated mouse (1:5000), goat (1:5000), and rabbit IgG (1:5000) (Santa Cruz,CA) as secondary antibodies. The target protein was detected with enhanced chemiluminescence Western blotting detection reagents (PIERCE). Membranes were stained with Ponceau-Red to confirm equivalent loading of total protein in all lanes. $\beta$-tubulin antibody (mouse; SIGMA-ALDRICH, Italy $1: 10.000$ ) was used to confirm proteins loading.

\section{Cell cycle analysis}

Asynchronously growing cells were transfected with $60 \mathrm{nM}$ MAD2 siRNA for $72 \mathrm{~h}$ before being pulse labeled with $10 \mu \mathrm{M}$ bromodeoxyuridine (BrdU) for the last $3 \mathrm{~h}$ posttransfection. Cells were fixed and stained with anti-BrdUFITC antibody, to detect S-phase cells, and propidium iodide (PI) to assess the DNA content and analyzed by flow cytometry. Labeling with BrdU allowed monitoring of cells actively engaged in DNA synthesis. DNA content was determined by treating the cells with PBS containing $4 \mu \mathrm{g} / \mathrm{mL}$ of PI and $40 \mu \mathrm{g} / \mathrm{mL}$ RNase. BrdU-labeled cells were analyzed as previously described (Barra et al., 2012; Lentini et al., 2012) with by FACSCanto (Becton Dickinson). Experiments were repeated at least twice and $>10,000$ events were analyzed by using the FACSDiva software.

\section{Determination of ploidy}

Cells were transfected with the specific siRNA (siGFP, siMAD2, siMAD2/siBUBR1) for $72 \mathrm{~h}$ treated with $0.2 \mu \mathrm{g} / \mathrm{mL}$ colcemid (Demecolcine, SIGMA-ALDRICH, Italy) and $1 \mathrm{~h}$, harvested by trypsinization, swollen in $75 \mathrm{mM} \mathrm{KCl}$ at $37^{\circ} \mathrm{C}$, fixed with 3:1 methanol/acetic acid (v/v), and dropped onto clean, ice-cold glass microscope slides. The slides were airdried and stained with a 3\% GIEMSA solution in phosphatebuffered saline for $10 \mathrm{~min}$. Chromosome numbers were counted using a Zeiss Axioskop microscope at a magnification of $100 \times$ objective.

\section{Trypan blue exclusion test of cell viability}

Cells were transfected with the specific siRNAs (siGFP, siMAD2, siBUBR1, siMAD2/siBUBR1) for $72 \mathrm{~h}$, harvested by trypsinization and collected in a tube with $4 \mathrm{~mL}$ of phosphatebuffered saline (PBS). Cell suspension $(100 \mu \mathrm{L})$ were mixed with $100 \mu \mathrm{L}$ of Trypan Blue (Sigma-Aldrich, Italy) and $10 \mu \mathrm{L}$ were placed in a Burker chamber for counting.

\section{Immunofluorescence microscopy}

To visualize $\beta$-tubulin, cells were grown on round glass coverslips and fixed with ethanol/acetic acid 95:5 for $10 \mathrm{~min}$, permeabilized with $0.01 \%$ TritonX (Sigma-Aldrich, Italy) in PBS for $15 \mathrm{~min}$ and blocked with $0.1 \%$ BSA for $30 \mathrm{~min}$ at room temperature. Coverslips were incubated with a mouse monoclonal antibody against $\beta$-tubulin mouse (SigmaAldrich, Italy, diluted 1:200) overnight at $4^{\circ} \mathrm{C}$, followed by a goat anti-mouse IgG-FITC secondary antibody (SIGMAALDRICH, Italy, diluted 1:200) for $1 \mathrm{~h}$ at $37^{\circ} \mathrm{C}$. Nuclei were stained with $1 \mu \mathrm{g} / \mathrm{mL} \quad 4^{\prime}, 6$-diamidino-2-phenylindole (DAPI) and examined on a Zeiss Axioskop microscope equipped for fluorescence; 200 nuclei for sample were analyzed and images were captured with a CCD digital camera (AxioCam, Zeiss) and printed by Adobe PhotoShop.

\section{Results}

\section{Co-depletion of MAD2 and BUBR1 by RNAi reduces cell} proliferation

We partially depleted MAD2 and BUBR1 (crucial components of the SAC) by RNA interference in HCT116 cells to investigate if a pathway $\mathrm{p} 53$ controlled is activated also in 
near-diploid tumor cells with a MIN phenotype to avoid propagation of highly aneuploidy cell. Partial reduction of both mitotic proteins was necessary to avoid deleterious effects on cell viability, since their complete depletion can induce death (Michel et al., 2001). Cells were transfected with specific synthetic siRNAs targeting MAD2 and BUBR1 transcripts (siMAD2, siBUBR1) and unspecific siRNA targeting the green fluorescent protein (siGFP) as a control. siRNAs targeting MAD2, BUBR1 and GFP transcripts were transfected individually or combined (siMAD2/siBUBR1, siMAD2/siGFP). Real-Time RT-PCR analysis showed decreased levels of MAD2 and BUBR1 transcripts in transfected HCT116 cells, demonstrating the efficacy of MAD2 and BUBR1 post-transcriptional silencing by RNAi (Figure 1A). In comparison to siGFP cells, siMAD2 and siBUBR1 cells expressed $\sim 60 \%$ MAD2 and 50\% BUBR1 transcripts, respectively (Figure 1A).

Western blot analyses confirmed reduction of Mad2 and Bubr1 protein levels in siMAD2 and siBUBR1 cells in comparison to siGFP cells (Figure 1B). At $72 \mathrm{~h}$, posttranscriptional silencing of MAD2, there was a fourfold increase of BUBR1 mRNA and protein expression levels
(Figure 1A-B). After BUBR1 posttranscriptional silencing MAD2 expression was reduced (Figure $1 \mathrm{~A}-\mathrm{B}$ ), possibly due to the slowdown of the cell proliferation.

Early effects $(72 \mathrm{~h}$ post-transfection) of MAD2 and BUBR1 partial post-transcriptional silencing resulted in slowing down of the cell proliferation of HCT-siMAD2 and HCT-siBUBR1 cells, as indicated by estimation of the cellular density/dish that was lower than the one of siGFP cells (Figure 1C, D). Reduction of proliferation was specific for cells transfected with siRNAs targeting MAD2 and BUBR1. In contrast, transfection with unspecific siRNA (siGFP) did not affect cell proliferation (Figure 1C, D). Trypan blue staining showed that double silenced HCTsiMAD2/siBUBR1 cells had higher mortality (about $80 \%$ ) in comparison to HCT-siMAD2 (40\%), HCT-siBUBR1 (50\%), and the double silenced HCT-siMAD2/siGFP cells (40\%).

Depletion of MAD2 and BUBR1 induces aneuploid cells and mitotic spindle alterations

To estimate the number of aneuploid cells after MAD2, BUBR1, and MAD2/BUBR1 post-transcriptional
A

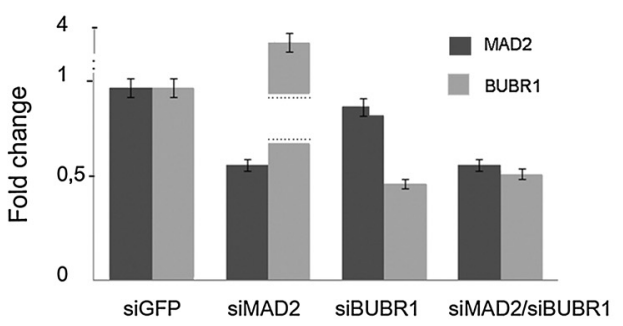

C

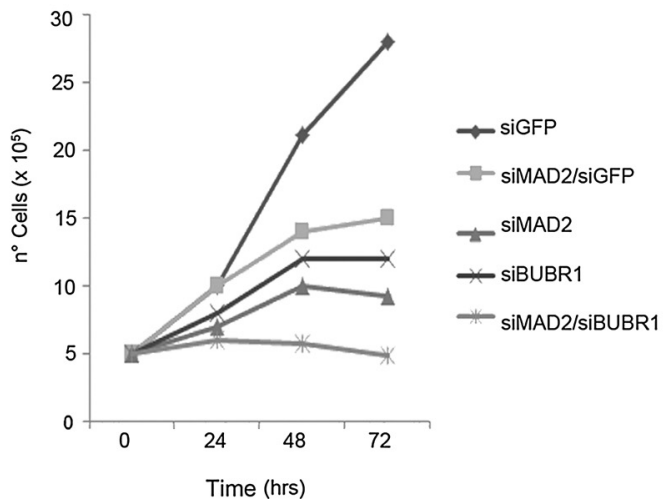

B
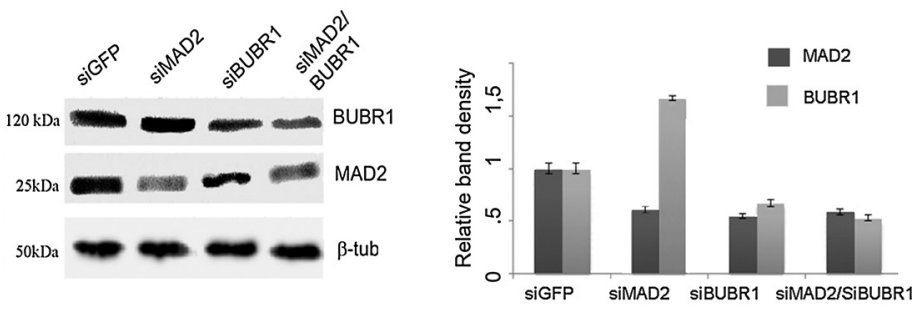

D

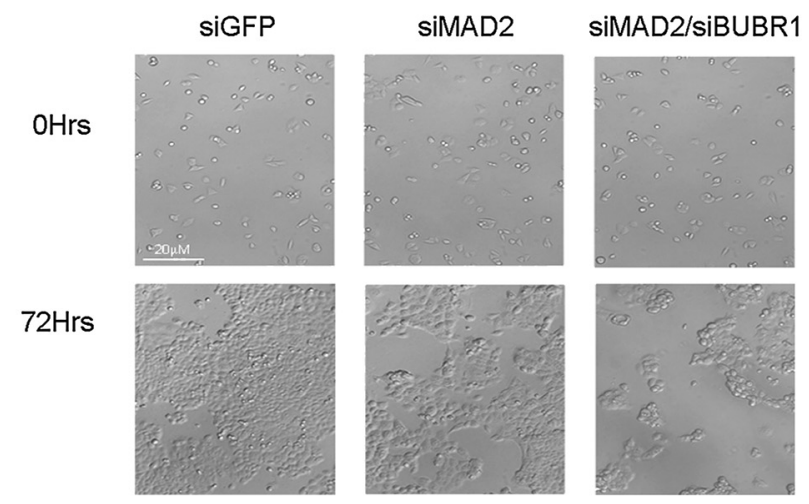

Figure 1 MAD2 and BUBR1 reduction by RNAi affects cell proliferation and viability. (A) Real-time RT PCR of HCT116 cells after $72 \mathrm{~h}$ from transfection shows reduced levels of both MAD2 and BUBR1 transcripts. (B) Western blot confirmed the reduction of MAD2 and BUBR1 expression also at protein level. The graph on the right shows increase in protein levels assessed by densitometry. (C) Graph of cell proliferation at 0-24-48, and $72 \mathrm{~h}$ post-transfection showing a delay in siMAD2/siBUBR1 transfected cells. (D) Images showing the morphology of transfected cells at 0 and $72 \mathrm{~h}$. Double silencing siMAD2/siBUBR1 transfected cells showed the presence of dead cells (round fragmented cells). 
A

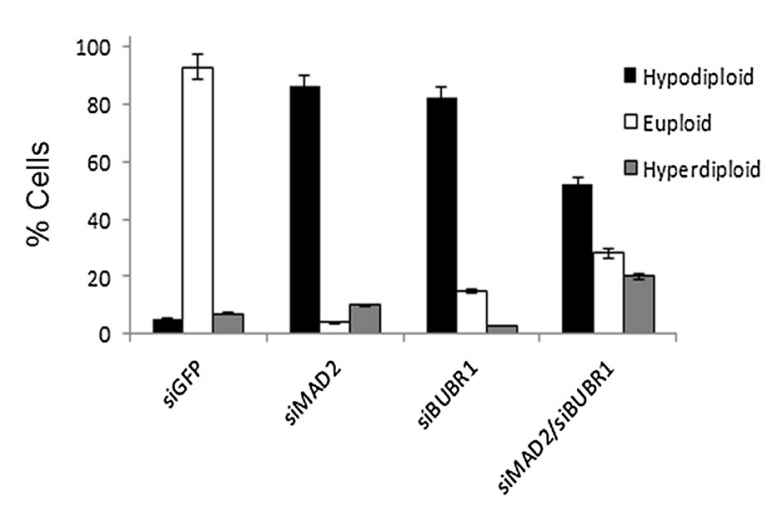

C

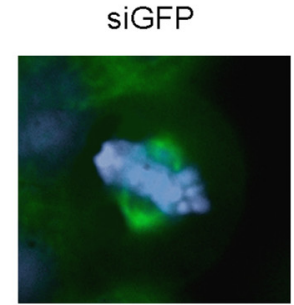

siMAD2
B
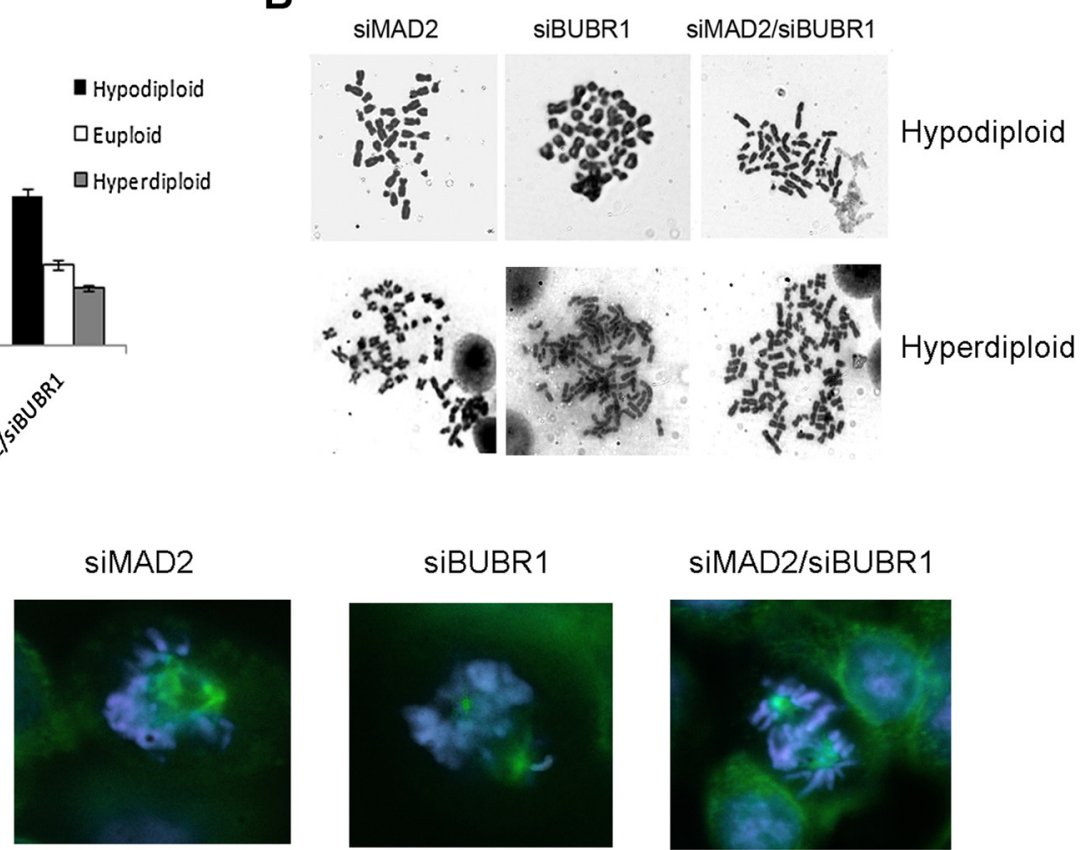

siBUBR1

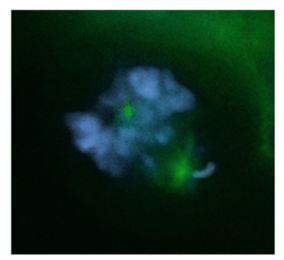

siMAD2/siBUBR1

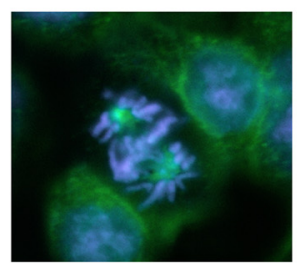

Figure 2 MAD2 and BUBR1 HCT116 depleted cells show aneuploidy, spindle alterations, and chromosome misalignment. (A) Histogram shows percentages of both aneuploid and diploid metaphases in HCT116 cells transfected with siRNA duplexes to repress MAD2 and BUBR1. Results were from three independent experiments (100-200 cells each) and bars indicate standard errors from the mean. (B) Images of representative GIEMSAstained aneuploid metaphases of siMAD2, siBUBR1, and siMAD2/siBUBR1 transfected cells exposed to colcemid. (C) Example of abnormal mitotic cell with spindle alterations and incorrect chromosome anchorage to the spindle in HCT116 cells after $72 \mathrm{~h}$ from transfection with the indicated siRNAs (siGFP, siMAD2, siBUBR1, siMAD2/siBUBR1). Cells were stained with DAPI to detect chromosomes (blue) and $\beta$-tubulin (green).

silencing, cells were treated with colcemid. The great majority of HCT-siMAD2 and HCT-siBUBR1 aneuploid cells (Figure 2A-B) were hypodiploid (85 and $82 \%$, respectively) and the less extent of hyperdiploids (10 and $3 \%$, respectively), whereas co-depleted HCT-siMAD2/ siBUBR1 cells had $18 \%$ hyperdiploid cells, $52 \%$ hypodiploid cells, and $30 \%$ euploid cells (Figure 2A-B). Immunofluorescence microscopy analyses by $\beta$-tubulin staining of HCT-siGFP, HCT-siMAD2, HCT-siBUBR1, and HCT-siMAD2/siBUBR1 indicated cells with mitotic spindle alterations as associated with incorrect distribution of chromosomes (Figure $2 \mathrm{C}$ and Figure $\mathrm{S1}$ ). Various mitotic spindle defects after MAD2, BUBR1, and MAD2/ BUBR1 post-transcriptional silencing were seen. In particular, monopolar spindles in $14 \%$ siMAD2, $12 \%$ siBUBR1, and $12 \%$ of siMAD2/siBUBR1 depleted cells, disorganized chromosomes in the spindle in $46 \%$ siMAD2, $48 \%$ siBUBR1, and $56 \%$ of siMAD2/siBUBR 1 silenced cells, respectively (Figure S1). In contrast, only $10 \%$ of HCT-siGFP cells showed alterations of mitotic spindle, which suggests that depletion of Mad2 and Bubr1 proteins individually or combined induced alterations of both chromosomes and the mitotic spindle.
MAD2 and BUBR1 co-depleted cells undergo p53dependent cells cycle arrest

The slowing down of proliferation after MAD2, BUBR1 and MAD2/BUBR1 post-transcriptional silencing (Figure 1C-D) suggests that part of these cells arrested or proceeded more slowly in the cell cycle. We therefore assessed by bivariate (PI and BrdU) FACScan (cytofluorimetric) analyses whether MAD2, BUBR1 and MAD2/BUBR1 post-transcriptional silencing was associated to different cell cycle profiles in HCT116 cells. Cells transfected with siRNA duplexes targeting MAD2, BUBR1, MAD2/BUBR1, and GFP were analyzed after $72 \mathrm{~h}$ from transfection and BrdU was added for $1 \mathrm{~h}$ to assess their ability to enter S-phase (Figure $3 \mathrm{~A}$ ). FACScan analyses was conducted only on adherent cells and showed that HCT-siMAD2 and HCT-siBUBR1 accumulated in G1 ( 82 and $86 \%$, respectively) and G2/M (10 and 6\%, respectively); only $8 \%$ of these cells were in S-phase (BrdU positive). In contrast, $14 \%$ of the HCT-siGFP control cells entered S-phase. As expected, only a minor fraction of HCTsiMAD2/siBUBR1 cells were in S-phase (BrdU positive: $0.9 \%)$ whereas all the remaining cells arrested in G1/S (87\%) and G2/M (12.1\%) (Figure 4A). 
A

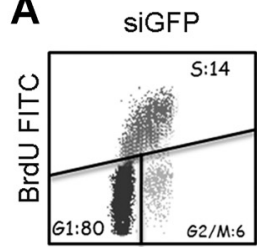

DNA content

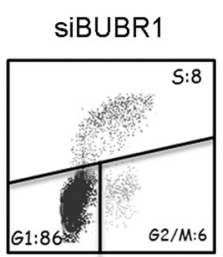

C

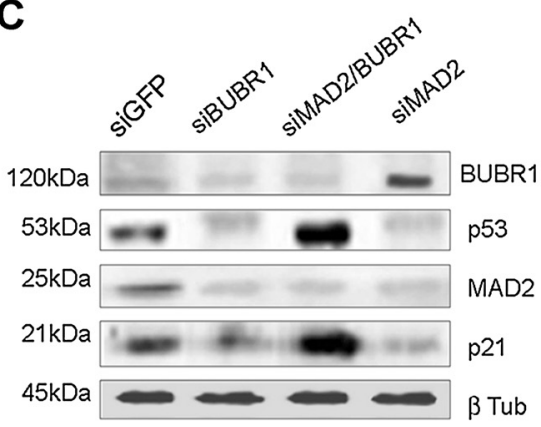

B

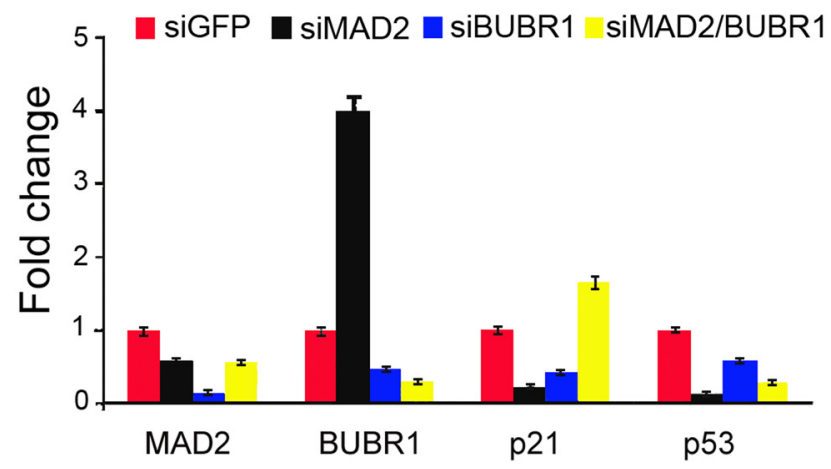

D

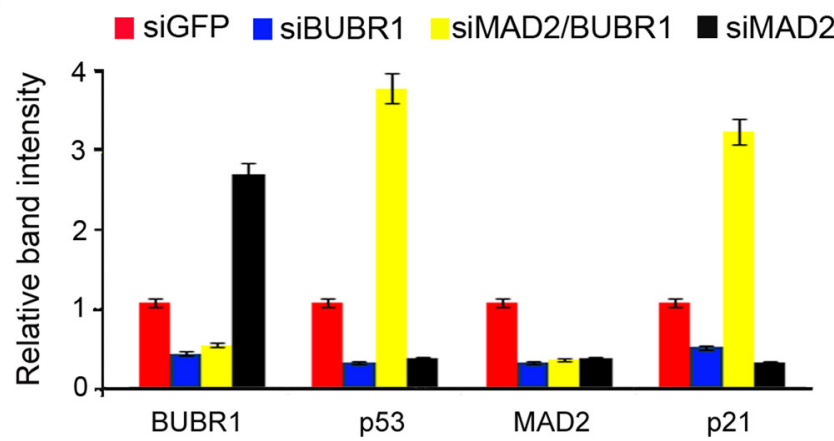

Figure 3 MAD2 and BUBR1 co-depleted cells underwent p53-dependent cells cycle arrest. (A) Cytofluorimetric profiles of HCTsiMAD2 HCTsiBUBR1 showing reduction of S-phase cells in respect to HCT-siGFP cells. On the contrary, double-silenced (siMAD2/siBUBR1) cells arrested in G1/S. DNA was stained with propidium iodide and S-phase by BrdU incorporation. (B) Real-time RT-PCR at $72 \mathrm{~h}$ post transfection shows BUBR1 and p21 waf1 increased levels in HCT-siMAD2 and HCT-siMAD2/siBUBR1, respectively. (C-D) Western blot analysis confirmed increases of BUBR1 in HCT-siMAD2 and p53 stabilization associated with an increase in HCT-siMAD2/siBUBR1, respectively. The graph on the right shows increase of protein levels assessed by densitometry.

Early effects of siMAD2/siBUBR1 post-trascriptional silencing on cell proliferation in HCT116 cells could be the result of the activation of a cellular G1/S checkpoint. Thus expression of the principal genes involved in G1/S checkpoint were investigated by Real-Time PCR and Western blot analysis. Increased levels of $\mathrm{p} 21^{\text {wafl }}$ transcript and protein in siMAD2/siBUBR1-HCT cells were seen (Figure 3B-D). The increased level of $\mathrm{p} 21^{\text {wafl }}$ that may have been induced by $\mathrm{p} 53$ seem to be stabilized, as indicated by its protein level (Figure 3C, D). To understand whether the p53 and $21^{\text {waf1 }}$ axis was responsible for the observed cell cycle arrest in siMAD2/siBUBR1-HCT cells, RNAi MAD2 and BUBR1 genes in combination with the p53 gene were silenced, and reduction of the MAD2, BUBR1, and p53 transcripts and protein assessed by RT-PCR and western blotting (Figure 4A, B). Flow cytometry showed that siMAD2/siBUBR1/siP53-HCT cells could re-enter in the cell cycle after $72 \mathrm{~h}$ from siRNAs transfection (Figure $4 \mathrm{C}$ ). siMAD2/siBUBR1/sip53-HCT cells also had a greater proliferative capacity than siMAD2/siBUBR1-HCT cells
(Figure 4D). However, there was no reduction of $\mathrm{p} 21^{\text {waf1 }}$ mRNA and protein levels after p53 depletion (Figure 4A, B). Similar results were obtained in cells null for p53; in fact transfected MAD2/BUBR1 siRNAs in HCT-p53KO cells proliferated better than HCT-siMAD2/BUBR1 cells after $72 \mathrm{~h}$ of transfection (Figure S2A-B). The findings suggest that HCT116 cells activate a p53-dependent cell cycle arrest and death (p21 $1^{\text {wafl }}$ independent) to prevent aneuploid cells propagation caused by depletion of MAD2 and BUBR1.

\section{Discussion}

Epidemiological and genetic analyses have led to an estimation of about six mutations being needed for malignant transformation on the somatic mutation theory of cancer (Vogelstein, 1996). Given the mutation rate in humans, it is extremely rare for a single cell to acquire all the necessary mutations. Thus, it has been postulated that destabilizing the genome is necessary for cancer development. 
A

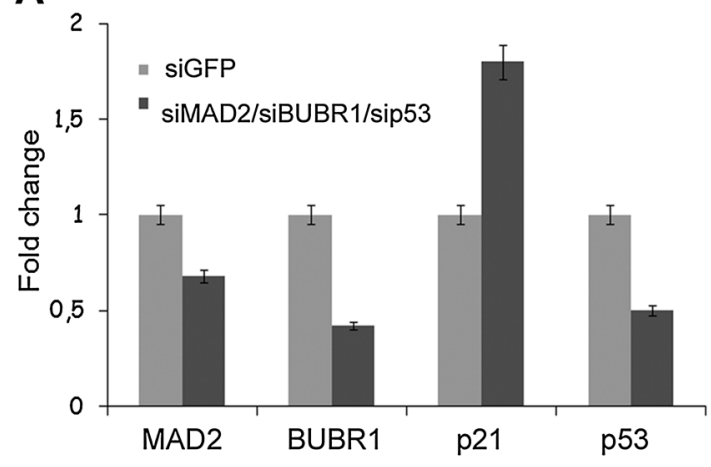

C

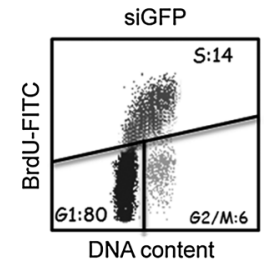

siMAD2/siBUBR1

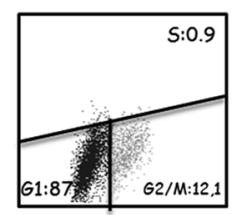

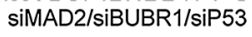

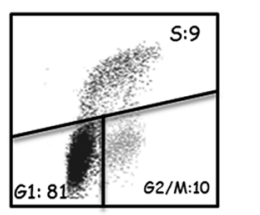

B

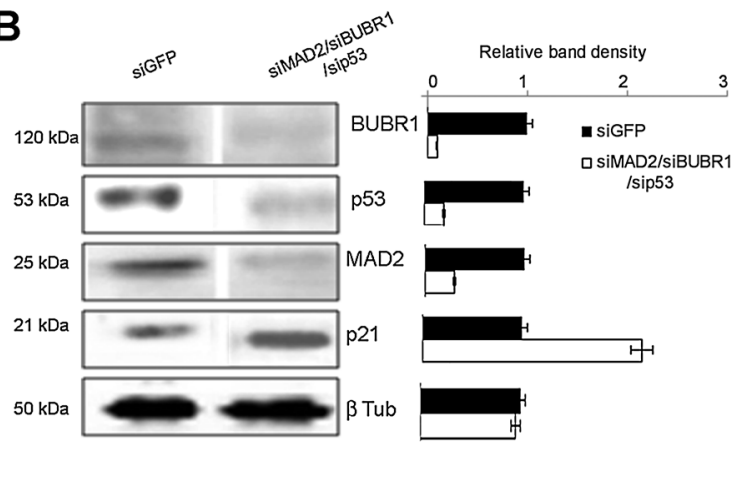

D

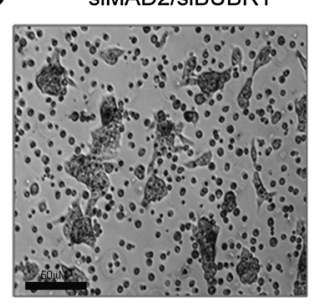

siMAD2/siBUBR1/siP53

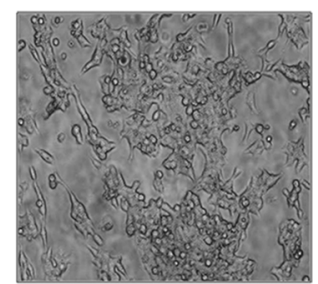

Figure 4 P53 silencing induces the re-enter in cell cycle of HCT- siMAD2/siBUBR1 cells (A) Real-time RT PCR shows that in the triple silenced (siMAD2/siBUBR1/siP53) cells p21 waf1 expression was similar of that in double (siMAD2/siBUBR1) silenced cells, and that p53 mRNA reduction was $\sim 50 \%$. (B) Western blot analysis showing that p53 was very low after triple silencing (siMAD2/siBUBR1/siP53), whereas p21 waf1 was always more expressed that in control cells. (C-D) Cytofluorimetry showing that triple-silenced (siMAD2/siBUBR1/siP53) cells re-enter cell cycle (presence of S-phase cells) and less dead cells (in suspension) in culture.

A prominent form of genetic instability in cancer is chromosomal instability (CIN) that results in gains or losses of whole chromosomes or translocation of chromosome segments. Alterations in chromosome number (aneuploidy) are found in nearly all tumor types of human origin. Aneuploidy would represent an epiphenomenon of cancer pathogenesis, a by-product of the hypermutable state exploited by tumor cells during the process of tumor progression to accumulate the mutant alleles essential for malignant proliferation (Zimonjic et al., 2001). However, no specific advantages can be associated with a particular gain or loss of a chromosome most of the time. Previously, we showed that is not sufficient to induce chromosomal instability in colon cancer cells with a MIN phenotype by destabilizing the mitotic spindle (for example by centrosome amplification); it has to be associated with alterations in genes regulating mitosis progression, such as AuroraA/STK15 to trigger CIN (Lentini et al., 2007).

Cancer cells can have gross abnormalities in their chromosome numbers, most as a result of defects in the quality control of sister chromatid separation (Weaver et al., 2007). Thus, a complete lack of SAC function does not cause cell lethality per se; rather, the cells are eliminated by other protecting mechanisms. Enhanced proteasomal degradation has been suggested as one aneuploidy tolerating mechanisms (Torres et al., 2010). Also, dysfunction of the
p38-P53 axis has been suggested as promoting proliferation of aneuploid human tumor cells (Burds et al., 2005). It was also suggested that heightened energy metabolism in aneuploid cells may be behind the surge in ROS levels. Increased ROS cause DNA damage that activates the ATM/P53 pathway, and this depends on the severity of aneuploidy (Li et al., 2010).

Simultaneous reduction of MAD2 and BUBR1 gene expression compromises cell viability and are consistent with previous observations showing that the simultaneous depletion of SAC genes could be exploited as a possible strategy to kill cancer cells (Burds et al., 2005). Furthermore, we suggest the weakening of the SAC checkpoint caused by MAD2 and BUBR1 reduction increased chromosome segregation errors, which in turn induced aneuploidy followed by arrest of cell proliferation and death associated with p53 stabilization. Following MAD2 transcript reduction there was a strong increase of the BUBR1 mRNA and protein expression levels. This is consistent with what already reported (Shin et al., 2003; Kops et al., 2004) and it could be correlated with a response to the aneuploidy (hyperdidploidy) induced by MAD2 depletion (Baker et al., 2013). Thus, BUBR1 seems to have an important role against aneuploid cells formation/propagation and its increase could be necessary to eliminate hyperdiploid cells from the population. The simultaneous reduction of the 2 SAC proteins induced high percentage of hyperdiploid 
cells and p53 stabilization, suggesting that hyperdiploidy could activate a p53 controlled pathway accordingly to published reports (Thompson and Compton DA:, 2010; Stuabach, in press). On the contrary, aneuploid cells generated by single silencing of MAD2 or BUBR1 did not show high levels of hyperdiploidy and did not activate the p53-controlled checkpoint. This conclusion is supported by p53 post-transcriptional silencing by siRNA in MAD2 and BUBR1

co-depleted cells allowing cells to re-enter the cell cycle. This was also seen in cells null for p53 (HCT-p53KO), when MAD2 and BUBR1 were simultaneously silenced. After p53 depletion by RNAi, we unexpectedly did not find a decrease of $\mathrm{p} 21^{\text {waf1 }}$ protein levels, which suggests that $\mathrm{p} 21^{\text {waf1 }}$ is only partially involved in the arrest of the cell cycle observed in HCT116-MAD2/BUBR1 cells and that its increase in aneuploidy cells is not only under the p53 control.

In conclusion, our results indicate that MAD2 and BUBR1 reduction causes mitotic spindle dis-organization and incorrect distribution of chromosomes along the spindle in the absence of mitotic poisons, and, importantly, that a p53-controlled pathway is required to induce cell cycle arrest or cell death to avoid aneuploid cells proliferation.

\section{Acknowledgments}

This work was partly supported by a grant (ex 60\%) from University of Palermo to ADL.

\section{Conflict of interest}

The authors declare that they have no competing interests.

\section{References}

Amato A, Lentini L, Schillaci T, Iovino F, Di Leonardo A (2009) RNAi mediated acute depletion of retinoblastoma protein (pRb) promotes aneuploidy in human primary cells via micronuclei formation. BMC Cell Biol 10: 79.

Aylon Y, Oren M (2011) P53: guardian of ploidy. Mol Oncol 5(4): 315-23.

Baker DJ, Dawlaty MM, Wijshake T, Jeganathan KB, Malureanu L, van Ree JH, Crespo-Diaz R, Reyes S, Seaburg L, Shapiro V (2013) Increased expression of BubR1 protects against aneuploidy and cancer and extends healthy lifespan. Nat Cell Biol 15(1): 96-102.

Barra V, Schillaci T, Lentini L, Costa G, Di Leonardo A (2012) Bypass of cell cycle arrest induced by transient DNMT1 posttranscriptional silencing triggers aneuploidy in human cells. Cell Div 7(1): 2.

Burds AA, Lutum AS, Sorger PK (2005) Generating chromosome instability through the simultaneous deletion of Mad2 and p53. Proc Natl Acad Sci USA 102(32): 11296-301.
Choi E, Lee H (2008) Chromosome damage in mitosis induces BubR1 activation and prometaphase arrest. FEBS Lett 582(12): 1700-6.

Frame FM, Rogoff HA, Pickering MT, Cress WD, Kowalik TF (2006) E2F1 induces MRN foci formation and a cell cycle checkpoint response in human fibroblasts. Oncogene 25(23): 3258-66.

Fukasawa K (2005) Centrosome amplification, chromosome instability and cancer development. Cancer Lett 230(1): 6-19.

Guo Y, Kim C, Ahmad S, Zhang J, Mao Y (2012) CENP-Edependent BubR1 autophosphorylation enhances chromosome alignment and the mitotic checkpoint. J Cell Biol 198(2): 205-17.

Hernando E, Nahle Z, Juan G, Diaz-Rodriguez E, Alaminos M, Hemann M, Michel L, Mittal V, Gerald W, Benezra R (2004) $\mathrm{Rb}$ inactivation promotes genomic instability by uncoupling cell cycle progression from mitotic control. Nature 430(7001): 797-802.

Iovino F, Lentini L, Amato A, Di Leonardo A (2006) RB acute loss induces centrosome amplification and aneuploidy in murine primary fibroblasts. Mol Cancer 5(1): 38.

Kops GJ, Foltz DR, Cleveland DW (2004) Lethality to human cancer cells through massive chromosome loss by inhibition of the mitotic checkpoint. Proc Natl Acad Sci USA 101(23): 8699704.

Lentini L, Pipitone L, Di Leonardo A (2002) Functional inactivation of $\mathrm{pRB}$ results in aneuploid mammalian cells after release from a mitotic block. Neoplasia 4(5): 380-7.

Lentini L, Iovino F, Amato A, Di Leonardo A (2006) Centrosome amplification induced by hydroxyurea leads to aneuploidy in $\mathrm{pRB}$ deficient human and mouse fibroblasts. Cancer Lett 238(1): 153-60.

Lentini L, Amato A, Schillaci T, Di Leonardo A (2007) Simultaneous Aurora-A/STK15 overexpression and centrosome amplification induce chromosomal instability in tumour cells with a MIN phenotype. BMC Cancer 7-212.

Lentini L, Barra V, Schillaci T, Di Leonardo A (2012) MAD2 depletion triggers premature cellular senescence in human primary fibroblasts by activating a p53 pathway preventing aneuploid cells propagation. J Cell Physiol 227(9): 3324-32.

Lentini L, Melfi R, Di Leonardo A, Spinello A, Barone G, Pace A, Palumbo Piccionello A, Pibiri I (2014) Toward a rationale for the PTC124 (Ataluren) promoted readthrough of premature stop codons: a computational approach and GFP-reporter cellbased assay. Mol Pharm.

Li M, Fang X, Baker DJ, Guo L, Gao X, Wei Z, Han S, van Deursen JM, Zhang P (2010) The ATM-p53 pathway suppresses aneuploidy-induced tumorigenesis. Proc Natl Acad Sci USA 107(32): 14188-93.

Matsuura S, Matsumoto Y, Morishima K, Izumi H, Matsumoto H, Ito E, Tsutsui K, Kobayashi J, Tauchi H, Kajiwara Y (2006) Monoallelic BUB1B mutations and defective mitotic-spindle checkpoint in seven families with premature chromatid separation (PCS) syndrome. Am J Med Genet A 140(4): 358-67. 
Michel LS, Liberal V, Chatterjee A, Kirchwegger R, Pasche B, Gerald W, Dobles M, Sorger PK, Murty VV, Benezra R (2001) MAD2 haplo-insufficiency causes premature anaphase and chromosome instability in mammalian cells. Nature 409(6818): 355-9.

Schvartzman JM, Duijf PH, Sotillo R, Coker C, Benezra (2011) $\mathrm{Mad} 2$ is a critical mediator of the chromosome instability observed upon $\mathrm{Rb}$ and p53 pathway inhibition. Cancer Cell 19(6): 701-14.

Shin HJ, Baek KH, Jeon AH, Park MT, Lee SJ, Kang CM, Lee HS, Yoo SH, Chung DH, Sung YC (2003) Dual roles of human BubR1, a mitotic checkpoint kinase, in the monitoring of chromosomal instability. Cancer Cell 4(6): 483-97.

Sotillo R, Hernando E, Diaz-Rodriguez E, Teruya-Feldstein J, Cordon-Cardo C, Lowe SW, Benezra R (2007) Mad2 overexpression promotes aneuploidy and tumorigenesis in mice. Cancer Cell 11(1): 9-23.

Stuabach AE (2004) The regulation of BubR1 expression by p53: a role for p53 in the mitotic spindle checkpoint and chromosome instability. Thesis submitted to the Division of Research and advanced studies of the University of Cincinnati.

Thompson SL, Compton DA (2010) Proliferation of aneuploid human cells is limited by a p53-dependent mechanism. J Cell Biol 188(3): 369-81.

Thompson SL, Compton DA (2008) Examining the link between chromosomal instability and aneuploidy in human cells. J Cell Biol 180(4): 665-72.

Torres EM, Williams BR, Amon A (2008) Aneuploidy: cells losing their balance. Genetics 179(2): 737-46.
Torres EM, Dephe N, Panneerselvam A, Tucker CM, Whittaker CA, Gygi SP, Dunham MJ, Amon A (2010) Identification of aneuploidy-tolerating mutations. Cell 143(1): 71-83.

Ulrich T (2012) Function of Lin9 in vivo and MAP3K4-p38 signaling regulates $\mathrm{p} 53$ mediated cell cycle arrest after defective mitosis. Dissertation of science doctoral degree the Bavarian Julius Maximilian University of Wurzburg.

Weaver BA, Silk AD, Montagna C, Verdier-Pinard P, Cleveland DW (2007) Aneuploidy acts both oncogenically and as a tumor suppressor. Cancer Cell 11(1): 25-36.

Zasadil LM, Britigan EM, Weaver BA (2013) 2n or not 2n: Aneuploidy, polyploidy and chromosomal instability in primary and tumor cells. Semin Cell Dev Biol 24(4): 370-9.

Zimonjic D, Brooks MW, Popescu N, Weinberg RA, Hahn WC (2001) Derivation of human tumor cells in vitro without widespread genomic instability. Cancer Res 61(24): 8838-44.

Received 7 January 2014; accepted 20 March 2014.

Final version published online 10 April 2014

\section{Supporting Information}

Additional supporting information may be found in the online version of this article at the publisher's web-site

Figure S1. Histogram showing the distribution of different mitotic alterations observed in wt and posttranscriptional silenced HCT116 cells.

Figure S2. MAD2 and BUBR1 reduction by RNAi did not affect cell proliferation and viability in HCT p53KO cells. 